\title{
Approximate solutions for the Skyrmion
}

\author{
J. A. Ponciano, L. N. Epele, H. Fanchiotti, and C. A. García Canal \\ Departamento de Física, Universidad Nacional de La Plata, C.C. 67 (1900) La Plata, Argentina
}

(Received 15 June 2001; published 20 September 2001)

\begin{abstract}
We reconsider the Euler-Lagrange equation for the Skyrme model in the hedgehog ansatz and study the analytical properties of the solitonic solution. In view of the lack of a closed form solution to the problem, we work on approximate analytical solutions. We show that Padé approximants are well suited to continue analytically the asymptotic representation obtained in terms of a power series expansion near the origin, obtaining explicit approximate solutions for the Skyrme equations. We improve the approximations by applying the two-point Padé approximant procedure whereby the exact behavior at spatial infinity is incorporated. An even better convergence to the exact solution is obtained by introducing a modified form for the approximants. The new representations share the same analytical properties with the exact solution at both small and large values of the radial variable $r$.
\end{abstract}

DOI: 10.1103/PhysRevC.64.045205

PACS number(s): 11.10.Lm, 12.39.Dc, 02.30.Mv

\section{INTRODUCTION}

The Skyrme model [1] provides a picture of baryons interacting via meson exchanges [2,3]. The model is based on the pre-QCD nonlinear $\sigma$ model, with the same group of chiral symmetry $G=S U\left(N_{f}\right) \times S U\left(N_{f}\right)$ [4]. Classical stability arguments require the presence of an additional term in the nonlinear $\sigma$-model Lagrangian [5]; this term was introduced initially by Skyrme. In this scenario, baryons emerge as solitonic solutions of the Euler-Lagrange equations [6], which are highly nonlinear second order equations and cannot be solved in exact closed form. Solutions are obtained by numerical integration. This is an awkward situation for the evaluation of physical quantities. In practice it is more convenient to have explicit approximate representations for the solitonic solutions.

In the present work we obtain reliable approximate representations for the solution of the Skyrme model in the $S U(2)_{L} \times S U(2)_{R}$ sector by means of the Padé approximant (PA) [7] procedure whereby the series expansion solution of the differential equations is continued analytically. The successful implementation of PA summation relies on the simple structure of the series solution near the origin which is almost an alternate geometric series. In order to reproduce the essential features of the exact solution at large distances, we use the two-point PA method and construct modified representations that share the exact properties of the Skyrmion configuration near both boundaries. Furthermore our approximate solutions are reliable in the whole interval of the independent variable $r$, namely $r=0$ to infinity.

This paper is organized as follows. In Sec. II we introduce the Skyrme model in the $S U(2)_{L} \times S U(2)_{R}$ sector and restrict the problem using the hedgehog ansatz. In Sec. III we present properties of the hedgehog solution showing power series representations for the chiral angle. In Sec. IV we introduce and use our approach based on the PA method. Finally, in Sec. V we draw the conclusions of our work.

\section{THE SKYRME MODEL}

Following Skyrme's proposal, baryons are soliton in the $\sigma$-nonlinear model with an additional stabilizing term. We will restrict ourselves to the sector $S U(2)_{L} \times S U(2)_{R}$ where the Lagrangian density of the model reads

$$
L=\frac{f_{\pi}^{2}}{4} \operatorname{Tr}\left(\partial_{\mu} U \partial^{\mu} U\right)+\frac{1}{32 g^{2}} \operatorname{Tr}\left[U^{+} \partial_{\mu} U, U^{+} \partial_{\nu} U\right]^{2},
$$

$U$ being a unitary operator. The parameter $f_{\pi}$ is the usual pion decay constant whose experimental value is $93 \mathrm{MeV}$ and $g$ is the dimensionless Skyrme parameter. Equivalently, $U$ can be expressed in terms of a scalar and a pseudoscalar field, $\sigma$ and $\pi$, respectively, in the following form:

$$
U=(\sigma+i \boldsymbol{\tau} \cdot \boldsymbol{\pi}) / f_{\pi} .
$$

It should be stressed that $\sigma$ and $\pi$ are not independent fields, and are related through $\sigma^{2}+\pi^{2}=f_{\pi}^{2}$. This restriction is the source of nonlinearities in the theory.

Rewriting $\boldsymbol{\pi}$ and $\sigma$ in terms of a new field $\boldsymbol{F}=f_{\pi} F \hat{\boldsymbol{\varphi}}$,

$$
\sigma=f_{\pi} \cos F, \quad \boldsymbol{\pi}=f_{\pi} \hat{\boldsymbol{\varphi}} \sin F,
$$

and going back to $U$, we obtain the representation

$$
U=\exp (i \tau \cdot F)
$$

Previous analysis [8] has shown that a reasonable agreement with experimental values of physical quantities is obtained when the hedgehog configuration of $U$ is adopted; this is a particular static ansatz where $\boldsymbol{F}$ takes a spherically symmetric form, namely,

$$
U_{s}(\tilde{r})=\exp [i \boldsymbol{\tau} \cdot \hat{\mathbf{n}} F(\tilde{r})]
$$

where $\hat{\boldsymbol{n}}$ denotes a unit radial vector.

In this representation, the energy reduces to a functional of the chiral angle, $F(\tilde{r})$, alone; 


$$
\begin{aligned}
E[F]= & 2 \pi \int_{0}^{\infty} d \tilde{r}\left[f_{\pi}^{2} \tilde{r}^{2}\left(\frac{d F}{d \tilde{r}}\right)^{2}+2 f_{\pi}^{2} \sin ^{2} F\right. \\
& \left.+\frac{1}{g^{2}} \sin ^{2} F\left(2\left(\frac{d F}{d \tilde{r}}\right)^{2}+\frac{\sin ^{2} F}{\tilde{r}^{2}}\right)\right] .
\end{aligned}
$$

The variation $\delta E / \delta F=0$ generates a nonlinear differential equation for $F(\tilde{r})$, whose solution corresponds to the static configuration of minimal energy. Written in terms of the dimensionless variable $r=g f_{\pi} \tilde{r}$, the differential equation for $F(r)$ reads

$$
\begin{aligned}
{\left[r^{2}+8 \sin ^{2} F(r)\right] \frac{d^{2} F(r)}{d r^{2}}=} & \frac{4}{r^{2}} \sin ^{2} F(r) \sin [2 F(r)] \\
& +\sin [2 F(r)]-2 r \frac{d F(r)}{d r} \\
& -4 \sin [2 F(r)]\left[\frac{d F(r)}{d r}\right]^{2}
\end{aligned}
$$

As finite energy solutions are required, $U(r)$ must tend to an arbitrary constant element of $S U(2)$ at spatial infinity. Choosing $U(r) \rightarrow 1$ as $r \rightarrow \infty$ implies the boundary condition

$$
F(\infty)=0
$$

This condition defines a mapping $S^{3} \rightarrow S^{3}$ from the compactified configuration space to the identity in the target space in $S U(2)$ which is isomorphic to $S^{3}$, falling into distinct equivalence classes labeled each by the winding number Z,

$$
\Pi_{3}[S U(2)]=\Pi_{3}\left(S^{3}\right)=Z \text {. }
$$

The integer number $Z$, which counts the coverings of the target space, is defined as the topological charge $q$ $=\int d x^{3} B_{0}$ related to the baryonic current

$$
B_{\mu}=\frac{1}{24 \pi^{2}} \epsilon_{\mu \nu \rho \sigma} \operatorname{Tr}\left[\left(U^{+} \partial^{\nu} U\right)\left(U^{+} \partial^{\rho} U\right)\left(U^{+} \partial^{\sigma} U\right)\right] .
$$

For the hedgehog ansatz, the baryon number charge density is

$$
B_{0}=-\frac{1}{2 \pi^{2}} \frac{d F}{d r} \frac{\sin ^{2} F}{r^{2}}
$$

and corresponds to a baryon number

$$
B=\frac{1}{2 \pi}[2 F(0)-2 F(\infty)-\sin 2 F(0)+\sin 2 F(\infty)] .
$$

From the foregoing, it follows that the solution belonging to the sector with baryon number $B=1$ satisfies the condition

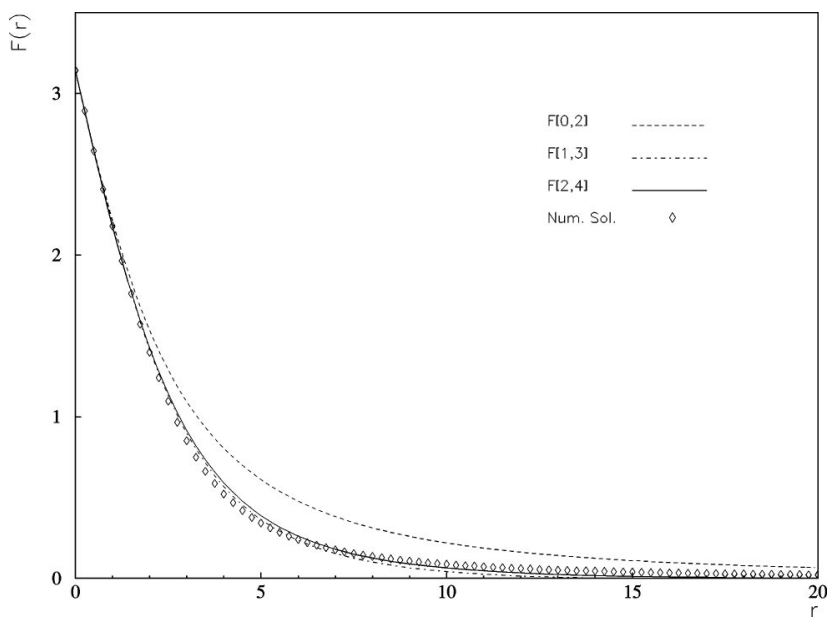

FIG. 1. Padé approximants to the hedgehog solution.

$$
F(0)=\pi
$$

The numerical solution labeled with $B=1$ is shown in Fig. 1 . Inserting this solution into Eq. (2.6) yields $E=23.2 \pi f_{\pi} / g$.

\section{PROPERTIES OF THE HEDGEHOG SOLUTION}

Before applying the PA procedure to the Skyrme model, we shall consider the solution of the Euler-Lagrange equation at both ends of the definition domain. Near the origin, a power series solution can be obtained [9],

$$
S_{H}(r)=\pi+F_{1} \tilde{r}+\frac{1}{3 !} F_{3} \tilde{r}^{3}+\frac{1}{5 !} F_{5} \tilde{r}^{5}+\ldots
$$

where $F_{1}$ turns out to be undetermined. All even powers have vanishing coefficients. Odd power coefficients are written in terms of $F_{1}$ and the dimensionless parameter $\phi$, defined as $\phi=F_{1} / g f_{\pi}$; for example,

$$
\begin{gathered}
F_{3}=-\frac{4}{5} F_{1}^{3} \frac{1+2 \phi^{2}}{1+8 \phi^{2}}, \\
F_{5}=\frac{24}{7} F_{1}^{5} \frac{1+(32 / 5) \phi^{2}+(88 / 5) \phi^{4}+(488 / 5) \phi^{6}}{1+24 \phi^{2}+192 \phi^{4}+512 \phi^{6}},
\end{gathered}
$$

while the results for higher terms may be found elsewhere [9]. In terms of the dimensionless variable $r=g f_{\pi} \tilde{r}$, expansion (3.1) becomes

$$
S_{H}(r)=\pi+\phi r+\frac{1}{3 !} \phi_{3} r^{3}+\frac{1}{5 !} \phi_{5} r^{5}+\cdots,
$$

where $\phi_{3}$ and $\phi_{5}$ are given by the same expressions as $F_{3}$ and $F_{5}$ but with $\phi$ instead of $F_{1}$. From now on, we shall be concerned only with the dimensionless variables.

For large values of $r$, there is a solution of the form $C / r^{2}$, as can be readily seen from trying this form in Eq. (2.7) and keeping only linear terms in $F(r)$. In order to find contribu- 
tions of higher order in $1 / r$, it is appropriate to perform the change of variable $\rho=1 / r$ in Eq. (2.7). In this new variable it reads

$$
\begin{aligned}
{\left[\rho^{2}+\right.} & \left.8 \rho^{4} \sin F(\rho)\right] \frac{d^{2} F(\rho)}{d \rho^{2}}+8 \rho^{3} \sin ^{2} F(\rho) \frac{F(\rho)}{d \rho} \\
& +4 \rho^{4} \sin ^{2}[2 F(\rho)]\left[\frac{d F(\rho)}{d \rho}\right]^{2} \\
& -4 \rho^{2} \sin ^{2} F(\rho) \sin [2 F(\rho)]-\sin [2 F \rho]=0 .
\end{aligned}
$$

Trying a power series expansion as before,

$$
F(\rho)=K_{0}+K_{1} \rho+\frac{1}{2 !} K_{2} \rho^{2}+\frac{1}{3 !} K_{3} \rho^{3}+\cdots,
$$

we find that the series solution contains just even powers. The first few coefficients written in terms of $K_{2}$ are [9]

$$
\begin{gathered}
K_{4}=0, \\
K_{6}=-\frac{30}{7} K_{2}^{3}, \\
K_{8}=-6720 K_{2}^{3} .
\end{gathered}
$$

Just as the initial slope $\phi$, the parameter $K_{2}$ cannot be determined from the differential equation. Approximate values for $\phi$ and $K_{2}$ are given in Ref. [9]. These values were determined through the use of integration routines based on shooting methods [4]. This is some sort of eigenvalue problem for $\phi$ and $K_{2}$. The solution is obtained by numerical integration of the differential equation, starting from the origin where the value of $\phi$ must be such as to satisfy the correct boundary condition corresponding to the relevant solitonic solution. Once $\phi$ is found, the value of $K_{2}$ is obtained in a similar way using Eq. (3.5). The reported values are [10]

$$
\phi=-1.0037, \quad K_{2}=17.2772 .
$$

These values, as well as the validity of the numerical solution can be checked in a consistent manner. We will not go on through this but just refer the reader to Ref. [11].

Let us make a slight modification in our analysis by rewriting the power expansion (3.4) in the following form

$$
S_{H}(r)=\pi+\phi r+a_{1} r^{3}\left(1+\frac{a_{2}}{a_{1}} r^{2}+\frac{a_{2}}{a_{1}} \frac{a_{3}}{a_{2}} r^{4}+\cdots\right),
$$

where the coefficients $a_{1}, a_{2}, a_{3}, \ldots$ are defined through

$$
a_{1}=\frac{\phi_{3}}{3 !}, \quad a_{2}=\frac{\phi_{5}}{5 !}, \quad a_{3}=\frac{\phi_{7}}{7 !},
$$

and so on. As shown in Table I, the numerical values of the ratios $-a_{n+1} / a_{n}$ vary very slowly with $n$. Thus, one can introduce the nearly constant $R \simeq-a_{n+1} / a_{n}$ (for any value of $n$ ). Consequently, the portion within the brackets in Eq.
TABLE I. Ratios of the coefficients of the series expansion (3.11).

\begin{tabular}{lllllll}
\hline$a_{2} / a_{1}$ & $a_{3} / a_{2}$ & $a_{4} / a_{3}$ & $a_{5} / a_{4}$ & $a_{6} / a_{5}$ & $a_{7} / a_{6}$ & $a_{8} / a_{7}$ \\
\hline-0.102 & -0.136 & -0.133 & -0.139 & -0.147 & -0.149 & -0.152
\end{tabular}

(3.11) can be replaced, to a good degree of approximation, by the alternate geometric series

$$
1-R r^{2}+\left(R r^{2}\right)^{2}-\left(R r^{2}\right)^{3}+\left(R r^{2}\right)^{4}-\cdots
$$

which has a radius of convergence $r_{0}$ defined by the relation $r_{0}^{2}=|1 / R|$.

Within the radius of convergence, $r \leqslant r_{0}$, expansion (3.11) may be replaced by

$$
\begin{aligned}
S_{H} \simeq & \pi+\phi r+a_{1} r^{3}\left[1-R r^{2}+\left(R r^{2}\right)^{2}-\left(R r^{2}\right)^{3}\right. \\
& \left.+\left(R r^{2}\right)^{4}-\cdots\right] .
\end{aligned}
$$

An alternate series such as $1-x+x^{2}-x^{3}+\cdots$ can be continued beyond its radius of convergence by the function (1 $+x)^{-1}$, so that $S_{H}$ can also be analytically continued for all positive values in the real $r$ axis using the representation

$$
F(r)=\pi+\phi r+\frac{a_{1} r^{3}}{1+R r^{2}} .
$$

Taking $R=-a_{2} / a_{1}$, Eq. (3.15) is exactly the [3,2] order Padé approximant representation of the series (3.11). The obvious task is to test whether a higher order of approximation can provide a better representation of the soliton. There is a great amount of examples [12] where the Padé approximant procedure provides a reliable analytic continuation of power series expansions and where PA representations converge rapidly to the relevant function of the problem. In the next section we will build different representations of the PA to the hedgehog solution showing that the features of reliability and fast convergence also hold in this problem.

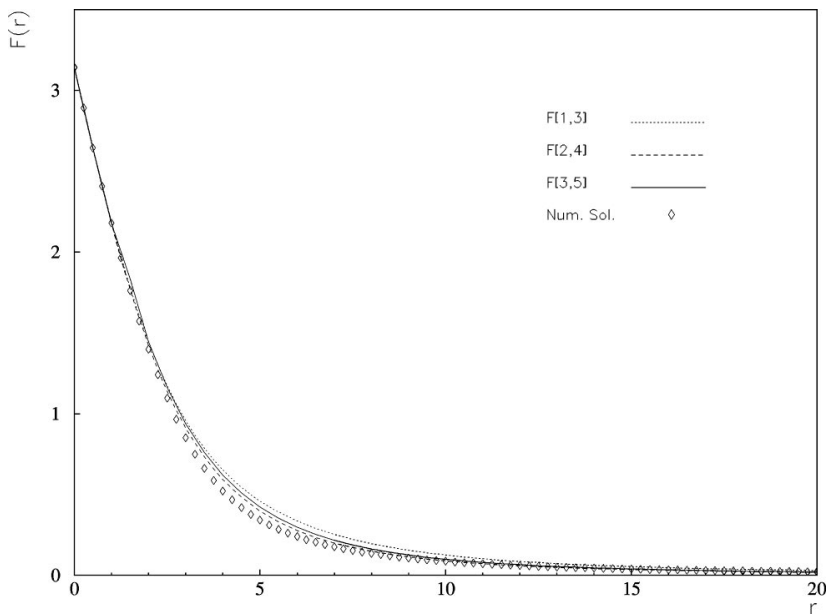

FIG. 2. Two-point PA to the hedgehog solution. 


\section{PADÉ APPROXIMANTS TO THE HEDGEHOG SOLUTION}

Our approach is based on Padé approximants, which are rational functions used to provide an analytic continuation of a power series representation of a given function, and are typically known to accelerate the convergence of the series. The Padé approximant $P_{[M, N]}(x)$ of order $[M, N]$ to the series $S(x)=\Sigma_{n} a_{n} x^{n}$ is defined as the ratio of two polynomials,

$$
P_{[M, N]}(x)=\frac{\sum_{k=0}^{M} A_{k} x^{k}}{\sum_{k=0}^{N} B_{k} x^{k}},
$$

where we set $B_{0}=1$ without loss of generality. The remaining $M+N+1$ coefficients are chosen so that the first $M$ $+N+1$ coefficients in the Taylor expansion of $P_{[M, N]}$ coincide with the series $S(x)$ through order $M+N$. Conversely, if only the first $M+N+1$ coefficients of the series $S(x)$ are known, such a Pade approximant can be used to predict the next coefficient in the series [13]

The PA of order $[M, N]$ to the hedgehog solution is found by comparing its Taylor expansion at the origin with series (3.1). From all the possible combinations $(M, N)$ allowed by the input series expansion (3.1), we are interested in those that may provide a suitable representation to the hedgehog

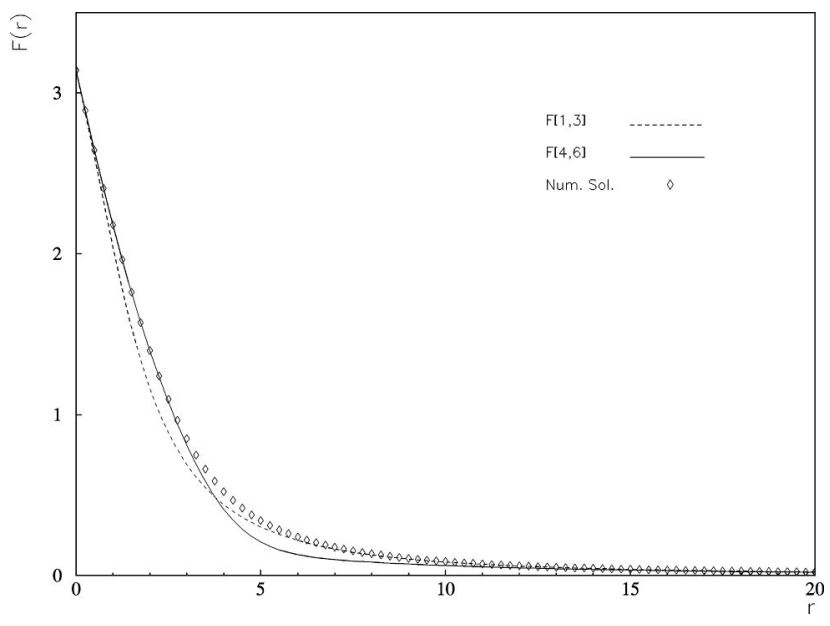

FIG. 3. Two-point PA exact up to order $O\left(1 / r^{3}\right)$ in the limit $r$ $\rightarrow \infty$.

solution. In order to enforce the conditions that fix the soliton solution to be of winding number one, we will require that the behavior at infinity of the PA representations is given by the leading term of expansion (3.6). To this end we impose the constraint $N-M=2$. We have computed the values of coefficients $a_{k}$ and $b_{k}$ corresponding to the sequence of PA's $[0,2],[1,3]$, and $[2,4]$ using the value $\phi_{1}=-1.003$ found by numerical methods. The results are displayed in Fig. 1. The explicit dependence of the PA's in terms of the coefficients of series (3.4) is

$$
\begin{gathered}
F_{[0,2]}=\frac{\pi}{1-\frac{\phi r}{\pi}+\left(\frac{\phi r}{\pi}\right)^{2}}, \\
F_{[1,3]}=\frac{\pi+\left(\phi+\frac{\phi \phi_{3} \pi^{2}}{6 \phi^{3}+\phi_{3} \pi^{2}}\right) r}{1+\frac{\phi \phi_{3} \pi}{6 \phi^{3}+\phi_{3} \pi^{2}} r-\frac{\phi^{2} \phi_{3}}{6 \phi^{3}+\phi_{3} \pi^{2}} r^{2}-\frac{\phi_{3}^{2} \pi}{6\left(6 \phi^{3}+\phi_{3} \pi^{2}\right)} r^{3}},
\end{gathered}
$$

for $[0,2]$ and $[1,3]$, respectively.

The PA representations built from the exact series solution near the origin do not guarantee the exact behavior at intermediate and large values of $r$. However, one may use the asymptotic solution given by the series (3.6) in order to find better representations of the hedgehog [14]. Yet, the right behavior at infinity may be incorporated using the two-point Padé approximant procedure. This is a natural extension of the usual PA method which consists in fixing almost one coefficient of the approximant using the asymptotic series solution at infinity [7]. We have computed the sequence of the two-point PA of orders $[1,3],[2,4]$, and $[3,5]$ (see Fig. 2). Certainly, the two-point PA representations are more reliable than the ordinary ones. The explicit form of PA $[1,3]$ is

$$
F_{[1,3]}=\frac{\pi+\frac{K_{2}\left(6 \phi^{3}+\phi_{3} \pi^{2}\right)}{6\left(K_{2} \phi^{2}-2 \pi^{3}\right)}}{1-\frac{\pi\left(K_{2} \phi_{3}+12 \phi \pi\right)}{6\left(2 \pi^{3}-K_{2} \phi^{2}\right)}-\frac{\phi\left(K_{2} \phi_{3}+12 \phi \pi\right)}{6\left(K_{2} \phi^{2}-2 \pi^{3}\right)}+\frac{6 \phi^{3}+\phi_{3} \pi^{2}}{3\left(K_{2} \phi^{2}-2 \pi^{3}\right)}} .
$$


The next step is to include the subleading behavior of the exact solution at infinity within the PA representations. We have done this up to order $O\left(1 / r^{3}\right)$ as shown in Fig. 3. As we go on with the procedure, the degree of approximation is improved both near the origin and for large values of $r$. However, for intermediate values of $r$, the PA representations are slowly convergent. Nevertheless we will show that there is a suitable functional form that provides very good representations to the hedgehog solution.

To this end, let us prescribe a modified PA of order $[0,4]$ through

$$
\widetilde{F}_{[0,4]}(r)=\frac{a}{\left(1+b_{1} r+b_{2} r^{2}+b_{3} r^{3}+b_{4} r^{4}\right)^{1 / 2}},
$$

such that the coefficients $a_{0}, b_{1}, b_{2}$, and $b_{3}$ are determined by expanding $\widetilde{F}_{[0,4]}(r)$ in a power series near the origin and matching the terms, order by order, with those of the exact solution (3.4) up to $O\left(r^{3}\right)$, and the remaining coefficient $b_{4}$ is fixed by using the leading term at infinity given by Eq. (3.6). We found explicitly,

$$
\widetilde{F}_{[0,4]}(r)=\frac{\pi}{\left(1-\frac{2 \phi}{\pi} r+\frac{3 \phi^{2}}{\pi^{2}} r^{2}+\frac{-12 \phi^{3}-\phi_{3} \pi^{2}}{3 \pi^{3}} r^{3}+\frac{4 \pi^{2}}{K_{2}^{2}} r^{4}\right)^{1 / 2}} .
$$

The form (4.4) can be generalized to higher orders $[0, k]$ as

$$
\widetilde{F}_{[0, k]}(r)=\frac{a}{\left(1+b_{1} r+b_{2} r^{2}+\cdots+b_{k} r^{k}\right)^{2 / k}},
$$

where the unknown coefficients may be found following the prescription described for the particular case $k=4$. We have built the representations corresponding to $k=6, k=8$, and $k=10$ (see Fig. 4). By construction, such representations have the exact Taylor expansion near the origin up to orders $O\left(r^{5}\right), O\left(r^{6}\right)$ and $O\left(r^{7}\right)$, respectively, as well as the exact leading behavior at infinity. The new PA representations improve in a remarkable way the representations obtained so far.

Following the program, an even better agreement for the large $r$ behavior may be obtained by including in the modified representations (4.6) the subleading behavior of the hedgehog solution. In Fig. 5 we have depicted the approximants so constructed. The figure shows an exceptional good

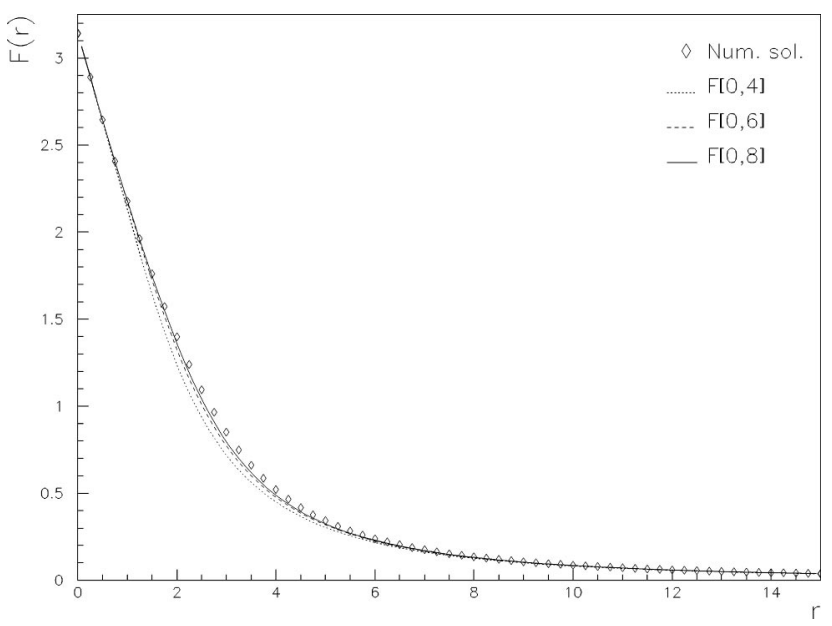

FIG. 4. Sequence of modified PA to the hedgehog solution. agreement between the numerical solution and the modified PA given by Eq. (4.6). In order to show the utility of the PA procedure as a method of analytic continuation, we have included in the same figure the power series representation near the origin up to and including order $O\left(r^{9}\right)$.

The utility of the PA method in this problem is outstanding, particularly for its simple and reliable application to the highly nonlinear Euler-Lagrange equation of Skyrme. Moreover, the PA representations reproduce exactly the main properties of the hedgehog solution at both small and large values of $r$.

In order to further check the reliability of the PA representations, we have calculated the corresponding baryon number given by

$$
B=-\frac{1}{2 \pi^{2}} \int_{0}^{\infty} \frac{d F}{d r} \frac{\sin ^{2} F}{r^{2}} 4 \pi r^{2} d r
$$

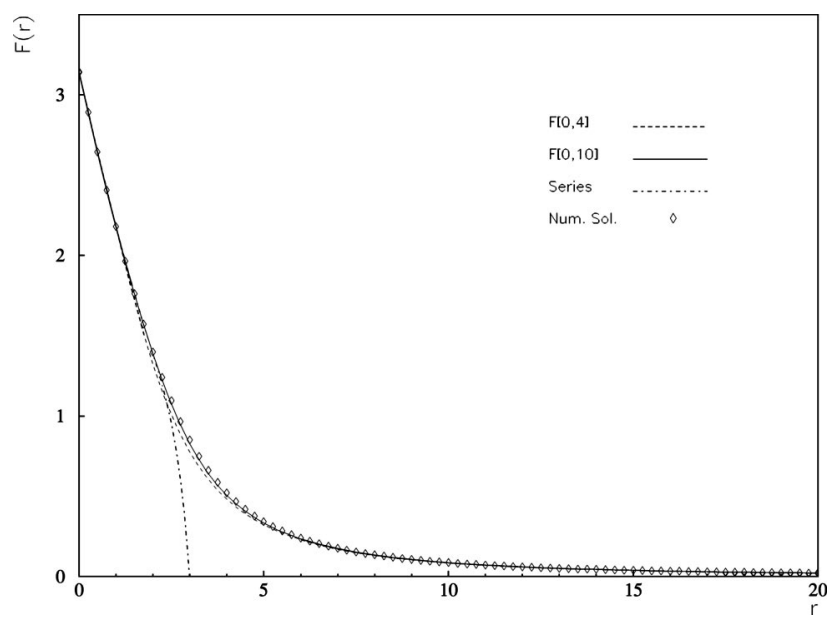

FIG. 5. Modified PA representations which reproduce the exact leading and subleading behavior at $r \rightarrow \infty$. 
and the minimal energy (2.6) whose numerical value is $E$ $=72.88 f_{\pi} / g$. We obtain $B=1$ for the two-point PA's which is exactly the topological number of the Skyrmion. As for the energy, the results for the last sequence of approximants in units of $f_{\pi} / g$ are $E_{[0,4]}=73.60, \quad E_{[0,6]}=73.02, \quad E_{[0,8]}$ $=72.96$, and $E_{[0,10]}=72.94$, which converge to the numerical value reported in previous works [4].

\section{FINAL REMARKS}

We have shown that the suggested PA representations for the Skyrme solutions can incorporate in a simple way the properties of the exact solution near both physical boundaries of the problem. This solves the difficulty in Ref. [11], where the authors assert the impossibility to obtain a PA to the series solution about the origin which, in addition, reproduces the exact behavior of the chiral angle at infinity. Moreover, our representations avoid the use of two separate ana- lytical representations for the Skyrmion, one for each of the two regions, which was the common approach to this problem [15], clearly unsuitable for phenomenological calculations.

Certainly, the results presented here highlight the utility of rational functions, the PA in particular, for analytic continuation of the asymptotic series. In this problem, the knowledge of the asymptotic behavior at two points favors a better convergence to the relevant function. The approximate analytical solutions can be used with reliability in the evaluation of physical quantities. The calculations are easier and more manageable than numerical computations.

\section{ACKNOWLEDGMENTS}

This research was supported in part by CONICET, Argentina. J.A.P. acknowledges MUTIS and CLAF for financial support.
[1] T.H. Skyrme, Proc. R. Soc. London, Ser. A 260, 127 (1961).

[2] E. Witten, Nucl. Phys. B223, 422 (1983); B233, 433 (1983).

[3] G.'t Hooft, Nucl. Phys. B72, 461 (1974).

[4] G.S. Adkins et al., Nucl. Phys. B228, 552 (1983).

[5] G.H. Derrick, J. Math. Phys. 5, 1252 (1964).

[6] R. Rajaraman, Solitons and Instantons-An Introduction to Solitons and Instantons in Quantum Field Theory (NorthHolland, Amsterdam, 1982).

[7] G.A. Baker, Jr. and P.R. Graves-Morris, Encyclopedia of Mathematics and its Applications (Cambridge University Press, London, 1981), Vols. 13 and 14.

[8] See, for example, A.P. Balachandran et al., Phys. Rev. D 27,
1153 (1983); M.P. Mattis and M.E. Peskin, ibid. 32, 58 (1985); M.P. Mattis and M. Karliner, ibid. 31, 2833 (1985).

[9] J.A. Mignaco and S. Wulk, J. Phys. G 18, 1309 (1992).

[10] A. Ebrahim and M. Savci, Phys. Lett. 166B, 173 (1986).

[11] J. Ananias et al., J. Math. Phys. 32, 1949 (1991).

[12] See, for example, J. Ellis et al., Phys. Lett. B 366, 268 (1996); G.A. Baker, Jr. and J.D. Johnson, Phys. Rev. A 44, 2271 (1991); L. N. Epele et al., ibid. 60, 280 (1999).

[13] M.A. Samuel and G. Li, Phys. Lett. B 331, 114 (1994).

[14] J. Linde and H. Snellman, J. Math. Phys. 33, 3740 (1992).

[15] M. Iwasaki and H. Ohyama, Phys. Rev. D 40, 3125 (1989); N. S. Manton and P. J. Ruback, Phys. Lett. B 181, 137 (1986). 